\title{
SUR LES BASES PHONÉTIQUES DES PRIMITIVES PHONOLOGIQUES ${ }^{\star}$
}

\author{
Ivaylo Burov \\ Département d'études romanes, Université de Sofia Saint Clément d'Ohrid, Sofia, Bulgarie
}

\section{Résumé}

Cet article s'interroge sur les bases phonétiques des théories traitant de la structure interne des segments. Il porte un regard sur la naissance et le développement de l'idée que le phonème n'est pas le constituant ultime de la chaîne phonique indissociable en unités plus petites, mais le produit de l'association d'une liste finie de paramètres universels appelés différemment par les divers modèles: traits, éléments, gestes. La rupture avec la conception « atomique » du phonème et l'élaboration des théories des primitives phonologiques vont cependant de pair avec une complication $\mathrm{du}$ formalisme et une hermétisation de la phonologie par son isolement de la phonétique.

\section{Mots-clés}

phonétique, phonologie, phonème, primitives phonologiques

\begin{abstract}
This paper questions the phonetic bases of the theories dealing with segments' internal structure. It takes a look at the rise and the development of the idea that the phoneme is not the ultimate constituent of the speech stream which cannot be broken down into smaller units, but the association product of a finite inventory of universal parameters called differently by different frameworks: features, elements, gestures. The break with the "atomic" conception of the phoneme and the elaboration of the phonological primitives theories go, however, hand in hand with a complicated formalism and with the hermetization of phonology by its estrangement from phonetics.
\end{abstract}

\section{Keywords}

phonetics, phonology, phoneme, phonological primitives

En tant que sciences de l'expression, au sens hjelmslévien du terme, la phonétique et la phonologie n'ont pas toujours été soigneusement distinguées et ont logiquement eu une histoire commune avant que l'objet d'étude et les méthodes de l'une et de l'autre aient été clairement délimités (Courtenay 1895, Troubetzkoy 1939 [1986]) et que chacune se soit engagée séparément dans une voie d'autonomisation à l'époque du structuralisme. Avec l'élaboration de plus en plus sophistiquée de l'appareil conceptuel de la phonologie et le développement parallèle des technologies d'analyse phonétique de la parole, les deux sciences ont

^ L'auteur tient à remercier sincèrement les évaluateurs de cet article pour l'aide apportée à sa rédaction française, ainsi que pour leurs remarques pertinentes et leurs suggestions utiles qui ont permis de mieux cerner la problématique traitée. 
commencé à s'isoler. L'isolement de la phonologie, focalisée sur ses propres représentations et usant d'une terminologie opaque, l'a rendue souvent difficile à comprendre pour les non-initiés, y compris pour les phonéticiens. Cette hermétisation, préparée par une vision algébrique et générative de la phonologie, trouve son point d'aboutissement dans la plupart des modèles post-génératifs nonlinéaires, ce que nous nous proposons d'illustrer dans cet article, en limitant notre attention à ceux d'entre eux traitant le problème des éléments constitutifs et de l'organisation interne des phonèmes. Nous n'évoquerons pas ici les modèles postgénératifs fixant leur analyse au niveau suprasegmental (Théories syllabiques et moraïques, Phonologie métrique, Phonologie prosodique) non seulement dans un esprit de concision et de cohérence, mais aussi pour deux autres raisons. Premièrement, c'est le problème de la nature du phonème et de ses propriétés pertinentes qui a permis de poser la phonologie comme science autonome et qui a préparé la grande explosion post-SPE, le modèle chomskyen ayant l'inconvénient de concevoir les propriétés prosodiques de la parole comme des propriétés segmentales. Deuxièmement, en parlant des éléments constitutifs des phonèmes, nombre de modèles segmentaux (Géométrie des traits, Théories des éléments, Phonologie articulatoire, etc.) font des références explicites à des notions relevant de la physique, de l'astronomie, de la chimie, de la biologie, lesquelles présentent un double intérêt pour l'histoire de la phonologie: d'une part, ces allusions rappellent le passé de la linguistique au $\mathrm{XIX}^{\mathrm{e}}$ siècle, considérée comme une science naturelle; d'autre part, elles témoignent peut-être d'une sorte de «complexe d'infériorité» par rapport aux sciences mûres et bien établies, ainsi que d'une volonté inconsciente d'asseoir la phonologie sur des fondements bien tangibles qu'elle semble en mal de se procurer dans son propre domaine. Au-delà d'une critique des excès du formalisme abstrait et des conceptions existantes des primitives phonologiques (qu'elles soient appelées traits, éléments ou gestes), cet article constitue aussi une tentative de réaffirmer et de démontrer le besoin d'une approche phonétique intégrée en vue d'expliquer la motivation des faits et des changements phonologiques.

\section{DÉBut DE LA FIN DE LA CONCEPTION «ATOMIQUE» DU PHONÈME}

Dès le milieu du $\mathrm{xx}^{\mathrm{e}}$ siècle, on assiste à l'émergence et à l'affirmation de la thèse que les systèmes phonémiques des langues résultent de la combinaison d'un nombre réduit de paramètres universels auxquels les divers modèles donnent des appellations différentes : traits, éléments, gestes, etc. Imposée par les travaux de Jakobson et ses collaborateurs (voir Jakobson, Fant et Halle 1952, Jakobson et Halle 1956, Jakobson 1963), cette idée sonne le glas de la conception «atomique» 
du phonème comme constituant ultime de la chaîne sonore, indécomposable en unités plus petites, défendue entre autres par Bloomfield (1935 [1973, p. 79]) et Troubetzkoy (1939 [1986, p. 44]).

Or, la conception du segment comme un complexe de propriétés différenciatrices se forge bien avant la naissance du structuralisme et du fonctionnalisme dans le Visible speech d'Alexander Melville Bell, publié en 1867. Dans cet ouvrage, celui-ci adopte pour la notation des sons attestés dans les langues du monde un alphabet phonétique universel contenant des symboles à caractère idéographique. Certains de ses symboles sont représentés dans la figure ci-dessous reproduite partiellement d'après Halle (2005, p. 27) :

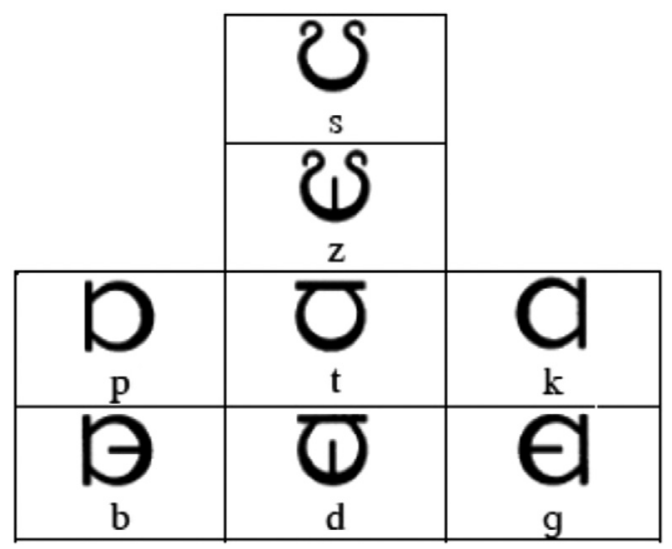

La figure (1) montre en effet que les consonnes sont représentées par une espèce de demi-cercle dont l'ouverture est orientée vers l'avant pour les labiales, vers le haut pour les coronales, et vers l'arrière pour les dorsales, ce qui reflète de manière originale le lieu d'articulation des trois ordres consonantiques. Dans le cas des plosives, l'orifice du demi-cercle est obstrué, alors que dans le cas des fricatives il est laissé ouvert. Par ailleurs, les voisées sont distinguées des non-voisées par un trait vertical se dressant depuis la base du demi-cercle.

Dans un article de 1911, le fils de Bell, Alexander Graham, l'inventeur du téléphone, affirme que les sons ne sont pas les atomes de la langue. À l'instar des composés chimiques, issus d'un mélange d'éléments du tableau périodique, les sons représenteraient d'après lui des combinaisons de constrictions et de mouvements effectués par les organes articulatoires (Halle 2005,p.26). Bloomfield (1935 [1973, p. 86]) note cependant que les symboles du Visible speech avaient l'inconvénient d'être difficiles à utiliser et d'induire des coûts d'impression élevés, raison pour laquelle ils se voient vite abandonnés et remplacés par les caractères de l'Alphabet phonétique international (API). Sa version de 1888 
est accompagnée de six principes directeurs relatifs à l'usage des caractères, dont voici ce que stipule le troisième : «L'alphabet sera autant que possible composé de lettres ordinaires de l'alphabet latin, en restreignant autant que possible l'utilisation de nouvelles lettres» (cité par Durand 2005a,p. 27). Ainsi, contrairement aux symboles de Bell, ceux de l'API ont un caractère alphabétique et ne reflètent pas les caractéristiques internes des segments. À ce sujet, Halle (2005, p. 27) indique que cette conception de l'API, «adoptée à l'origine pour des raisons d'économie financière », a eu finalement pour « conséquence malheureuse » de « rendre obscure et de vouer à l'oubli la découverte de Bell que les sons de la parole sont des complexes de traits». Cela explique pourquoi cette thèse fondamentale ne sera redécouverte que « 60 ans plus tard par Jakobson et ses collaborateurs ${ }^{1}$.

L'analyse du phonème en traits distinctifs est indispensable pour la formalisation des notions de classe naturelle, de corrélation ou de marque, ainsi que de divers processus phonologiques, mais Jakobson et ses collaborateurs ont essuyé des critiques pour avoir imposé un cadre théorique trop rigide, celui du binarisme, jugé contre-intuitif pour la phonétique. En effet, la matière phonétique n'est pas binaire ou catégorielle mais repose sur un continuum de mouvements articulatoires et de signaux acoustiques qui souvent n'identifient pas les phonèmes simplement par leur présence ou leur absence, mais aussi par leur netteté ou leur synchronisation les uns par rapport aux autres. Ainsi, Malmberg (1966, p. 169) évoque-t-il le fait que le suédois connaît deux voyelles hautes d'avant contrastant en degré de labialisation, ou plutôt en mode de labialisation à en croire les données radiographiques de Ladefoged et Maddieson (1996, p. 295-296), distinction dont il est difficile de rendre compte si l'on s'en tient au trait jakobsonien bémolisé/non bémolisé et si l'on se prive des oppositions graduelles posées par Troubetzkoy. Pour justifier le format des traits, Jakobson soutient que le binarisme serait au centre de l'activité humaine et que les cellules du cerveau fonctionneraient en mode binaire de façon à réduire le message à des prises de décisions alternatives du type oui/non. Mais Martinet (1955, p. 73-75) ne verra dans sa théorie qu'«un appareil physicomathématique [conçu] pour l'agrément des esprits avides de rigueur abstraite » et rejettera le binarisme avec l'argument qu'il ne repose pas sur des observations d'un corpus suffisamment large et représentatif de langues.

En réponse à ses critiques, Morris Halle publie un article au titre significatif, «In defense of the number Two » (Halle 1957), où il essaie de prouver que l'analyse binaire est simplement plus économique. On sait que c'est notamment avec lui et Noam Chomsky que le binarisme s'affirmera définitivement et se radicalisera, accusant ainsi le divorce entre phonétique et phonologie. 
La publication de Sound pattern of English (SPE, Chomsky et Halle 1968) s'inscrit en effet dans le modèle mentaliste de Chomsky (1957) qui rompt avec la tradition bloomfieldienne et distributionnaliste américaine, basée sur l'empirisme descriptiviste et le rejet de toute approche psychologique dans l'analyse linguistique. Chomsky n'a hérité de ses illustres prédécesseurs qu'un goût prononcé pour la formalisation mais non l'intérêt pour l'analyse de corpus d'énoncés réellement produits. Il ne s'intéresse à la performance des sujets parlants que dans la mesure où celle-ci peut renseigner sur leur compétence ou connaissance intuitive innée des structures profondes de la langue, seul véritable objet de la linguistique.

L'intérêt de Chomsky et Halle (1968) pour le mécanisme de génération de la substance phonique allait de pair avec une conception modulaire de la grammaire dont les différentes composantes sont reliées par des règles transformationnelles. La notion de transformation confère au modèle génératif un caractère foncièrement dérivationnel et dynamique mais contribue en même temps à la complication de son appareil théorique. En réalité, SPE voit la génération de l'énoncé comme un long processus de transformations successives au cours duquel une structure syntaxique profonde est convertie progressivement en une structure syntaxique de surface, cette dernière étant soumise à son tour à l'action de règles de réajustement, avant d'être transférée à la composante phonologique et traitée par une autre série de règles. Les règles phonologiques sont formulées à l'aide d'un nouvel inventaire de traits, toujours binaires mais en nombre relativement plus grand et définis en termes articulatoires, contrairement à ceux de Jakobson qui avait privilégié l'aspect acoustique de la parole pour leur formulation.

Bien des critiques ont été adressées à SPE: modèle peu plausible de la production et de la perception, impliquant la maîtrise et l'application de la part des locuteurs d'une longue série de règles compliquées dans un ordre strictement déterminé; marginalisation du lexique, considéré comme un ensemble non structuré d'unités lexicales présentant souvent un comportement imprévisible et idiosyncrasique; intégration mécanique de la morphologie à un niveau phonologique sous-jacent où des séparateurs morphémiques et lexicaux figurent à côté des phonèmes; vision linéaire de la phonologie dont découle un traitement de traits prosodiques comme des traits segmentaux; surpuissance des règles phonologiques que rien n'empêche d'engendrer des changements non attestés et invraisemblables, ou des segments anthropophoniquement impossibles.

Pour ce qui est des implications du nouveau format des traits binaires, force est de signaler quelques points de détail. Premièrement, Chomsky et Halle estiment pouvoir se passer du trait [vocalique], dont la fonction principale est de distinguer les voyelles des glides, en lui substituant le trait [syllabique]. Cette position leur vaudra les critiques de Fromkin (1970) et, en général, celles de la Phonologie 
autosegmentale, stipulant que la syllabicité d'un segment découle directement de sa position dans la syllabe (voir Clements et Keyser 1983, Kaye et Lowenstamm 1984). De même, la Phonologie métrique (voir notamment Liberman et Prince 1977, Selkirk 1984, Hayes 1995) s'oppose à l'idée que le trait [accentué] serait une propriété paradigmatique des segments, faisant valoir qu'un élément est perçu comme accentué dans le plan syntagmatique par son contraste avec les éléments voisins de la chaîne phonique. En effet, s'appuyant sur les thèses de Chomsky et Halle, plusieurs linguistes travaillant dans le cadre génératif considèrent à tort ces deux propriétés prosodiques comme des traits segmentaux, ce qui rend inefficace et arbitraire la formalisation de phénomènes conditionnés prosodiquement.

Deuxièmement, les traits chomskyens [haut], [bas] et [arrière], reflétant les mouvements du dos de la langue et correspondant, avec leur valeur positive, respectivement aux traits jakobsoniens diffus, compact et grave, reviennent à ignorer les oppositions graduelles de degré d'aperture introduites par Troubetzkoy et à radicaliser la tradition binariste de Jakobson. Cette radicalisation est amorcée déjà par Halle (1957) qui, dans l'intention de mieux capter le continuum allant des voyelles hautes aux voyelles basses, propose de scinder le registre compact/diffus originellement proposé en deux sous-registres : compact/non compact et diffus/non diffus. Or, la combinaison de ces deux registres ne permettant de distinguer que trois degrés de relèvement de la langue, les phonologues fidèles à la tradition générativiste se voient obligés de recourir au trait $[ \pm$ tendu $]$ ou à son substitut fréquemment utilisé $[ \pm \mathrm{ARL}]^{2}$ pour la description de systèmes à quatre ou à cinq degrés d'aperture. Toutefois, cette solution traduit mal l'intuition que les voyelles du tableau ci-dessous forment un continuum de hauteur vocalique et que certaines combinaisons de valeurs (et notamment $[+$ haut, + bas $]$ ) sont en effet impossibles.

(2)

\begin{tabular}{|l|c|c|c|c|c|c|c|c|c|}
\cline { 2 - 10 } \multicolumn{1}{c|}{} & i & I & e & $\varepsilon$ & a & o & o & u & u \\
\hline Haut & + & + & - & - & - & - & - & + & + \\
\hline ARL & + & - & + & - & - & - & + & - & + \\
\hline Bas & - & - & - & - & + & - & - & - & - \\
\hline
\end{tabular}

Il en va de même pour [arrière], dont les deux valeurs opposées sont inaptes à rendre compte de systèmes opposant des voyelles centrales et postérieures qui ne contrastent pas en labialisation. Clements (1993b, p. 107-108) note à ce sujet que les langues recourant à ce type d'oppositions sont rares mais loin d'être inexistantes, citant entre autres, les cas du suédois de Finlande, du tsou, du nemboi, du woléaien et du nimboran. 
Une autre conséquence indésirable de l'absolutisation du binarisme est le rejet des oppositions équipollentes posées par Troubetzkoy (1986) et identifiées, entre autres, avec celles de lieu d'articulation. Comme dans le formalisme génératif le point d'articulation des consonnes est indiqué principalement par les traits [ \pm antérieur], $[ \pm$ coronal] et $[ \pm$ arrière], désormais les plosives $/ \mathrm{p} / \mathrm{et} / \mathrm{t} /, \mathrm{par}$ exemple, ne s'opposeront plus comme une labiale à une coronale, mais comme une coronale à une non coronale (3), tout en s'intégrant dans la classe naturelle des consonnes antérieures produites «avant la zone palato-alvéolaire » (à en croire la définition de Chomsky et Halle 1968, p. 304). Comme le précise cependant Yip (1989, p. 350), des processus phonologiques affectant simultanément des labiales et des coronales antérieures (par exemple, $/ \mathrm{p}, \mathrm{f}, \theta, \mathrm{t}, \mathrm{s} /$, etc.), sans affecter en même temps des coronales non-antérieures (comme $/ \int, \mathrm{s}, 6 /$, etc.), ne semblent pas documentés dans la littérature. Dans ce sens, le trait [ \pm antérieur] s'avère inadéquat, dès lors qu'il instaure une distinction inconnue des systèmes phonologiques.

(3)

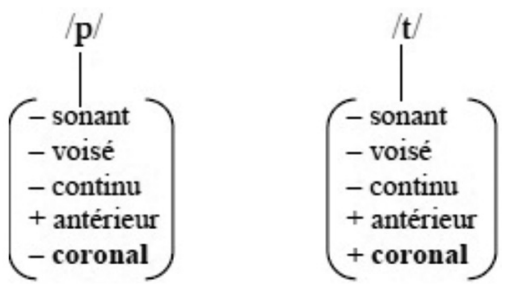

Au-delà des excès du binarisme, le développement du modèle génératif transformationnel contribue fortement à faire de la phonologie un domaine hermétique en l'arrachant à ses fondements phonétiques. Puisque le formalisme de SPE est supposé orienté vers la découverte de la réalité profonde ou mentale sousjacente à une réalisation phonétique concrète, ses règles, l'ordre de leur application et l'inventaire des traits commencent à être perçus comme pleinement suffisants pour expliquer les changements phonologiques sans que ceux-ci soient mis en rapport avec les paramètres physiques et physiologiques de l'appareil articulatoire. Malheureusement, ces critiques peuvent être adressées aussi à de nombreuses phonologies post-génératives qui, au lieu de chercher à combler le fossé entre phonétique et phonologie laissé par SPE, orientent leurs efforts vers la «sophistication» du formalisme abstrait.

\section{LES PHONOLOGIES POST-GÉNÉRATIVES ET L'ACHÈVEMENT DU FORMALISME}

Comme il a été déjà dit, une des critiques principales adressées au modèle chomskyen concerne sa vision linéaire de la phonologie qui fait que des traits segmentaux comme $[ \pm$ haut], $[ \pm$ voisé], $[ \pm$ nasal], etc. sont formellement indistincts de traits prosodiques tels que $[ \pm$ syllabique $]$ ou $[ \pm$ accentué $]$. C'est en réaction 
contre cette position qu'on voit apparaître au milieu des années 1970 deux grandes mouvances parallèles: la Phonologie métrique (Liberman 1975, Liberman et Prince 1977) et la Phonologie autosegmentale (Goldsmith 1976). Les deux ont en commun d'être non-linéaires ou multilinéaires, en ce sens qu'elles prévoient des plans séparés pour les dimensions segmentale, temporelle et prosodique de la parole. Elles ne sont pas des théories monolithiques mais plutôt des cadres relativement larges où s'inscrivent bon nombre de modèles qui acceptent certains de leurs postulats, tout en en réinterprétant d'autres. Quoique s'opposant à la phonologie générative, beaucoup de ces modèles s'appuient sur certains de ses principes de base : distinction entre structures profondes et superficielles, recours à des règles dérivationnelles, adoption d'un inventaire universel de primitives phonologiques (et même de traits binaires), conception modulaire de la grammaire, mise en rapport du formalisme avec la notion de marque, etc. Dans ce sens, ils sont plus post-génératifs qu'anti-génératifs. Comme nous ne pouvons envisager de présenter ici, fût-ce de façon sommaire, les formalismes qui se réclament de ces deux mouvances et conformément aux objectifs annoncés en introduction de l'article, nous n'évoquerons que les modèles fixant leur analyse sur le plan subsegmental. Dans les trois sous-sections qui suivent, ceux-ci seront groupés et examinés successivement selon qu'ils posent comme unités constitutives des phonèmes des traits (Géométrie des traits, théories de la sous-spécification), des éléments (Phonologie du charme et du gouvernement, Phonologie de dépendance, Phonologie des particules) ou des gestes (Phonologie articulatoire). Sans chercher à passer en revue tous les problèmes et enjeux posés par les nombreuses versions de ces modèles, nous chercherons plutôt à dégager ce qui, au-delà des dénominations génériques différentes, constitue la cohérence de ces trois visions de la nature des primitives phonologiques. Nous verrons notamment que la cohérence de chacune des trois mouvances apparaît entre autres dans trois types de métaphores courantes faisant allusion aux sciences naturelles et témoignant de la volonté des phonologies non-linéaires d'asseoir de façon mécanique leurs thèses sur des fondements extralinguistiques.

\subsection{Théories des traits}

Parmi les cadres qui adoptent et développent la position de Jakobson, Chomsky et Halle selon laquelle les primitives sont des traits, on distingue les modèles géométriques des modèles spécificationnels. Si les premiers émettent des hypothèses sur l'organisation structurale des traits et les rapports entre eux, les seconds s'intéressent surtout à la pertinence ou à la redondance de leurs valeurs pour la phonologie des langues. La Géométrie des traits (GT) (voir entre autres Clements 1985, 1993a, b, McCarthy 1988, Odden 1991, 1994, Clements et Hume 1995, Sagey 1986, Halle 1995, Halle, Vaux et Wolfe 2000) est fondée sur le 
principe que le phonème n'est pas simplement un ensemble de traits ordonnés de façon arbitraire sous forme de matrice, comme le soutenaient les structuralistes et les générativistes, mais un ensemble de complexes de traits, possédant une structure interne. Plus concrètement, les traits sont groupés en nœuds en fonction de critères articulatoires et fonctionnels, formant ainsi une arborescence dont les constituants et les ramifications sont subordonnés à un nœud majeur dénommé Racine pour rester dans la même imagerie. Voici, à titre illustratif, la géométrie des consonnes telle qu'elle est conçue notamment par Clements et Hume (1995, p. 292):

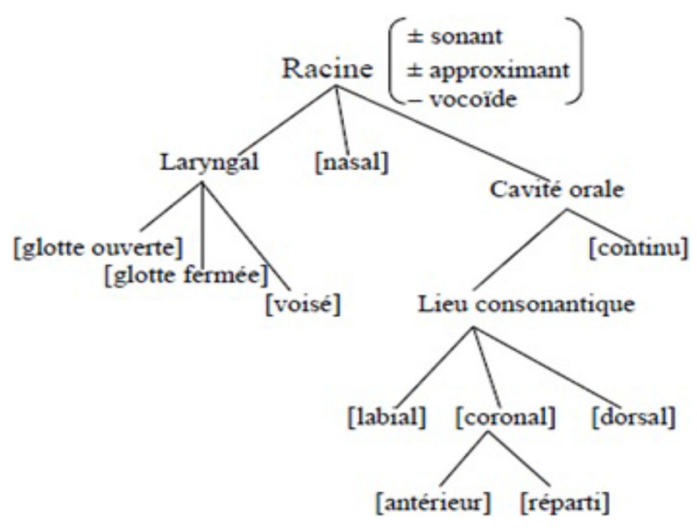

Les processus segmentaux agissent sur cette arborescence par dissociation, promotion et propagation de traits ou de nœuds entiers dans la hiérarchie. Par propagation d'un nœud on peut, entre autres, formaliser de façon naturelle et économique une des assimilations les plus courantes dans les langues, à la suite de laquelle une consonne nasale adopte le lieu d'articulation de l'obstruante suivante : [mp], [mf], [nt], [nk], etc. Sur ce point, le cadre préconisé par SPE s'était montré défaillant car ses règles transformationnelles ne pouvaient décrire le processus que de la façon suivante:

$$
\left(\begin{array}{l}
+ \text { consonantique } \\
+ \text { nasal }
\end{array}\right) \longrightarrow\left(\begin{array}{l}
\alpha \text { antérieur } \\
\beta \text { coronal } \\
\gamma \text { continu } \\
\delta \text { arrière }
\end{array}\right) /-\left(\begin{array}{l}
\alpha \text { antérieur̀ } \\
\beta \text { coronal } \\
\gamma \text { continu } \\
\delta \text { arière }
\end{array}\right)
$$

Or, comme l'indique Durand (2005b,p.76), une pareille formulation «n'exprime pas vraiment l'intuition que c'est le lieu d'articulation in toto qui s'assimile et non telle ou telle combinaison arbitraire de traits». C'est la raison pour laquelle la GT conçoit ce phénomène comme une propagation du nœud buccal de l'obstruante avec l'ensemble des ramifications qui lui sont subordonnées. Celui- 
ci vient s'associer avec la nasale précédente dont le lieu d'articulation n'est pas spécifié au niveau sous-jacent:

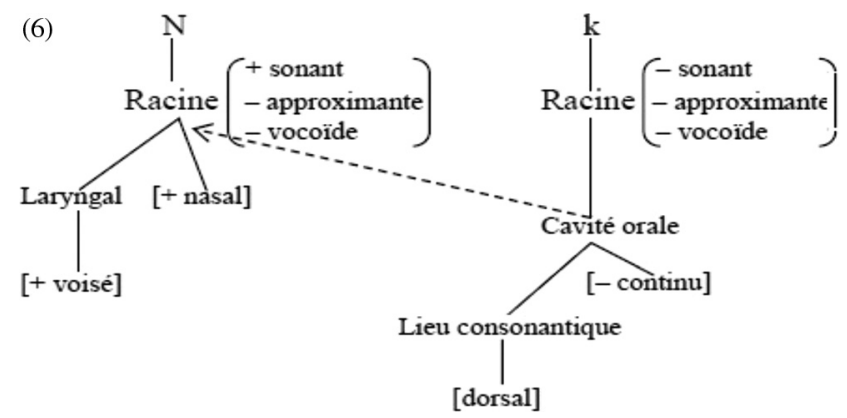

Le fait que cette assimilation soit largement diffusée à travers les langues constitue un argument en faveur de l'existence de structures intermédiaires entre les niveaux segmental et subsegmental, et notamment de complexes de traits regroupés selon des critères anatomiques. Cette thèse est confirmée également par les processus solidement attestés de débuccalisation, consistant en l'aspiration de fricatives (par exemple, /f, $\mathrm{s}, \mathrm{x} / \rightarrow / \mathrm{h} /$ ) ou en la glottalisation de plosives (par exemple, $/ \mathrm{p}, \mathrm{t}, \mathrm{k} / \mathrm{h} / \mathrm{R} /$ ). Comme l'indique son appellation même, la débuccalisation se traduit en GT par la perte de toutes les spécifications liées à l'activité des organes de la cavité orale, lors de laquelle les spécifications glottales sont préservées.

Comparée aux modèles structuralistes et générativistes du contenu des phonèmes, la GT apparait comme une théorie plus restrictive. D'après Clements (1985, p. 230), le principe hiérarchico-componentiel de structuration des segments est censé traduire l'interdépendance des mouvements effectués par les articulateurs ou leur relative autonomie: "the relative independence of any two features or feature classes is correlated with the number of nodes that separate them ». Ainsi, les traits reflétant les mouvements des organes de la cavité buccale sont-ils nettement émancipés sur le plan structural des traits laryngaux. Ce choix exprime l'idée que, malgré le nombre et la complexité des spécifications orales, celles-ci peuvent se propager « en bloc », comme en (6), sans affecter les traits dominés par les autres nœuds.

Or, les paramètres concrets des arborescences varient sensiblement d'une version de la GT à une autre. Ainsi, Clements (1985, p. 248) place-t-il sous le nœud de mode d'articulation les traits [nasal], [continu] et [strident]; chez Clements et Hume (1995) cependant, les deux premiers sont redistribués sous des nœuds différents, alors que le troisième est carrément supprimé (4). Les traits de lieu ont fait eux aussi l'objet de controverses : outre les spécifications [labial], [coronal] et [dorsal], Clements (1993b) introduit logiquement un quatrième trait de point 
d'articulation, à savoir [radical]; dans la mesure où il désigne l'emplacement de la constriction des voyelles basses et des consonnes pharyngales, celui-ci est appelé [pharyngal] par McCarthy (1988), Odden (1994) et Burov (2013). En même temps, certains auteurs misent sur une version plus ramifiée et hiérarchisée du nœud Lieu : Avery et Rice (1989, p. 195), par exemple, ne subordonnent pas [labial] et [dorsal] directement au constituant majeur indiquant le point d'articulation, mais à un constituant secondaire dénommé Périphérique. Gerlach (2010,p.28), elle, se déclare pour le regroupement de [coronal] et [dorsal] au sein d'un sous-constituant Lingual :

(7a)

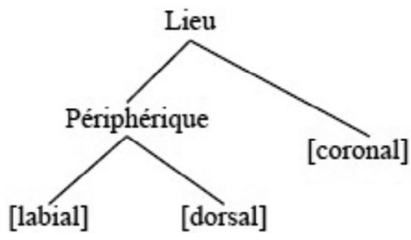

(7b)

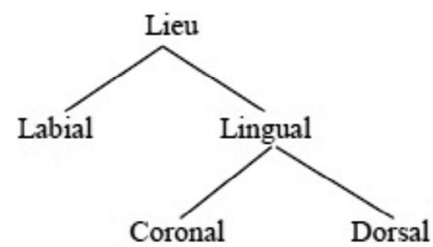

Pour ce qui est du degré d'aperture des voyelles, Clements (1993a) admet qu'il peut être représenté par un seul et même trait scalaire [ouvert], susceptible de se scinder en plusieurs « registres » : $\left[ \pm\right.$ ouvert $\left._{1}\right],\left[ \pm\right.$ ouvert $\left._{2}\right],\left[ \pm\right.$ ouvert $\left._{3}\right]$, etc., chacune des valeurs du trait étant disposée sur une ligne autosegmentale séparée et pouvant se propager de manière autonome. La solution de Parkinson (1996) va dans le même sens, à ceci près que le choix d'un trait d'aperture scalaire dénommé [fermé] a des implications tout à fait différentes sur la nature des assimilations partielles de hauteur vocalique. Odden (1991), enfin, préserve les traits standards [haut], [bas], et [ARL], tout en les englobant dans un même sous-constituant Hauteur, comme illustré ci-dessous :

(8)

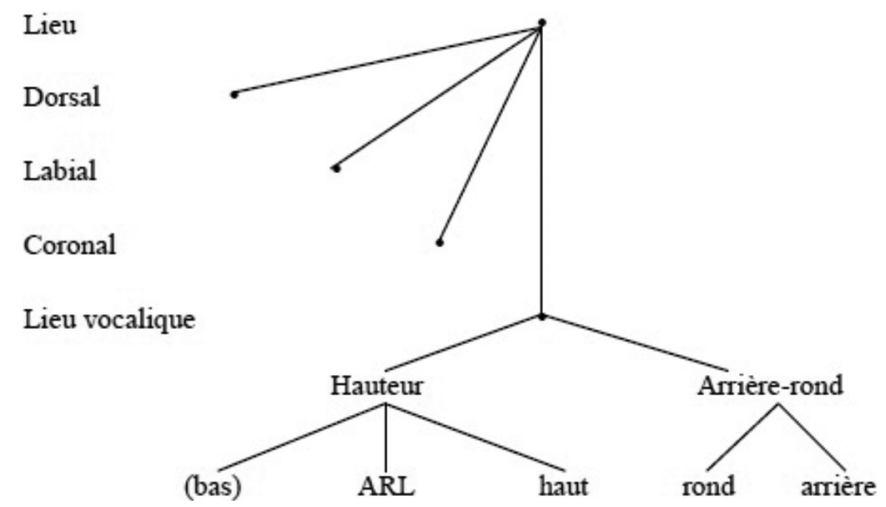


À l'encontre de toutes ces solutions, mais aussi des principes de la Théorie articulatoire révisée (défendue par Halle 1995 et Halle, Vaux et Wolfe 2000), Halle (2005) adopte une géométrie simplifiée à l'extrême où tous les traits sont directement dominés par le nœud Racine, sans être subordonnés à aucun constituant intermédiaire :

(9)

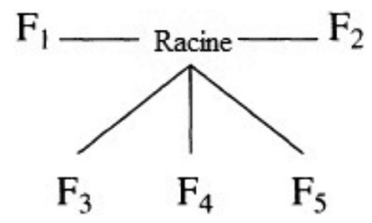

Les éléments terminaux en (9), étiquetés $F_{1}, F_{2}, F_{3}$, etc., peuvent représenter n'importe quel trait, qu'il soit de classe majeure ou articulatoire, mono- ou bivalent. Halle (2005, p. 36) illustre cela par la dérivation en (10), censé traduire le processus de "palatalisation», responsable d'après lui des alternances en anglais du type electri $[k] \sim$ elektri[s]ity, analo[g] analo[d3]y. L'opération schématisée cidessous montre plus concrètement comment la spécification coronale d'une voyelle d'avant vient se substituer à la spécification dorsale d'une consonne vélaire :

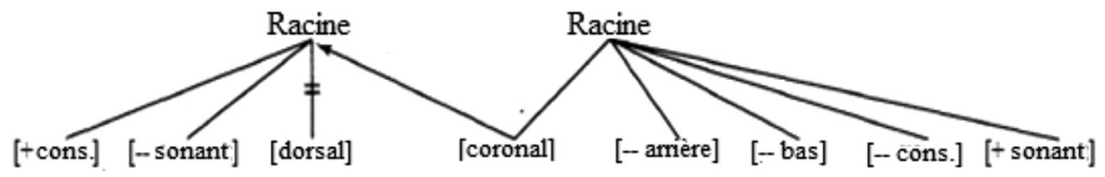

L'adoption d'une structure plus plate par la suppression de tout constituant intermédiaire et le traitement de tous les traits comme ayant le même statut formel reviennent cependant à rompre radicalement avec les principes de base de la GT. En outre, l'idée qui se cristallise chez Halle (2005) que n'importe quels traits peuvent être transférés et ajoutés ensemble ou séparément lors des processus phonologiques ramène d'un seul coup la phonologie des décennies en arrière à l'époque où le phonème était considéré comme une matrice de traits ordonnés de façon arbitraire. L'article en question ne permet pas de décider si cette position est un renoncement à la recherche d'une arborescence universelle dont les nœuds et les traits pourraient capter la spécificité de tous les processus phonologiques dans toutes les langues et posséder en même temps un pouvoir de prédiction. En réalité, le regroupement de certains traits sous une étiquette articulatoire présuppose que ces derniers devraient fonctionner comme une classe naturelle, mais l'interdépendance de deux ou plusieurs traits dans un processus concret et dans une langue donnée est vue 
souvent, au terme d'un raisonnement circulaire, comme un argument pour introduire un nœud universellement pertinent. Cela étant, la non pertinence de ce même nœud dans d'autres langues sera tout à fait attendue mais remettrait en cause l'omnivalidité des ramifications.

L'Hypothèse de la dépendance des traits (Mester 1986, D'Intorno et Weston 2000) constitue une alternative à l'universalité de la structure hiérarchicocomponentielle posée par les divers modèles géométriques. Comme l'indique son appellation même, elle admet que les traits entretiennent des rapports de dépendance, leur hiérarchie n'étant pas universellement fixée et immuable, mais variant selon les langues. Cela permet la formation de configurations ad hoc de traits et de lignes associatives selon les patrons segmentaux observés dans les langues, mais prive la théorie de portée généralisatrice et de pouvoir de prédiction, tout en réduisant son formalisme à une schématisation de la variation interlinguistique du comportement des traits. Or, ceci n'est pas nécessairement un défaut, s'il est vrai qu'une des tâches principales de la phonologie théorique est la «simplicité de la description» (Halle 1964). Même si l'on admet l'existence d'un inventaire universel de traits, il est tout à fait logique que les diverses langues n'en utilisent qu'un petit sous-ensemble pour la structuration de leurs systèmes segmentaux et qu'elles configurent différemment les rapports entre eux.

La hiérarchisation ad hoc des traits est moins contestable du point de vue phonologique que du point de vue phonétique, étant donné que tout modèle subsegmental doit reposer sur des bases articulatoires et/ou acoustiques solides. Selon Burov (2017), la subordination d'un trait à un autre est justifiable dans la mesure où les différents articulateurs sont reliés et le mouvement de l'un d'entre eux provoque souvent le déplacement d'un autre (l'abaissement du vélum, par exemple, est souvent une conséquence automatique de la constriction du pharynx). Or, dans les modèles de Mester (1986) et de D'Intorno et Weston (2000), il n'y a aucun obstacle formel à ce que n'importe quel trait soit dominé par n'importe quel autre, indépendamment de leurs corrélats phonétiques.

\subsection{Des traits aux éléments}

Les théories binaires ont l'inconvénient de ne pas pouvoir formaliser correctement la notion de segment possible à cause du grand nombre de traits dont elles se servent, chacun possédant une valeur positive ou une valeur négative, ce qui constitue un inventaire pouvant engendrer, en théorie, des combinaisons pratiquement infinies et, partant, des segments anthropophoniquement impossibles. Pour limiter ce potentiel excessif de génération, les théories des traits recourent souvent à des filtres ou à des règles de nettoyage qui laissent une impression d'arbitraire des changements phonologiques. C'est une des raisons pour lesquelles elles ont été déclarées périmées par les Théories des éléments (TE), 
appellation générique mettant sous un dénominateur commun trois mouvances proches conceptuellement: Phonologie de dépendance (Anderson et Ewen 1987, Durand 1990, etc.), Phonologie de gouvernement (Kaye, Lowenstamm et Vergnaud 1988, 1990, Harris 1996, Scheer 1999, etc.) et Phonologie des particules (Schane 1984, Carvalho 1993, etc.).

On peut considérer qu'au fond les TE radicalisent les postulats de base des Théories de la sous-spécification, qui ont deux versions principales : contrastive (Steriade 1987, Clements 1988) et radicale (Archangeli 1988, Archangeli et Pulleyblank 1994). La Théorie de la sous-spécification contrastive (TSC) repose sur la thèse que les valeurs de certains traits sont redondantes et prévisibles par rapport aux valeurs d'autres traits (par exemple, le voisement dans le cadre des sonantes ou l'arrondissement dans le cadre des voyelles postérieures non basses), ce qui rend inutile leur encodage dans le contenu phonologique des segments. Elles figurent dans le segment pour ainsi dire «sous forme latente» et ne se manifestent qu'au niveau phonétique, où tous les segments sont par définition pleinement spécifiés. Seuls les traits pertinents (ou contrastifs) sont censés figurer dans la représentation sous-jacente des segments. Quant à la Théorie de la sousspécification radicale (TSR), elle est une version plus rigide et extrême de la TSC, comme l'indique son appellation même. En tant que telle, elle est encore plus «parcimonieuse» en spécifications dans l'encodage du contenu des segments, lequel est censé se faire non seulement en fonction de la contrastivité/redondance mutuelle des traits, mais aussi en fonction de la notion de marque: seules les valeurs marquées sont encodées dans les représentations profondes, alors que les valeurs non-marquées sont ajoutées lors de la dérivation par des règles de redondance comme celles en $(11)^{3}$.

$$
\begin{aligned}
& \text { a) } \quad[\quad] \rightarrow[\text { nasal }] \\
& \text { b) } \quad[\quad] \rightarrow[\text { coronal }]
\end{aligned}
$$

En admettant qu'en principe aucun trait n'est binaire au niveau sous-jacent, puisqu'une seule de ses valeurs est accessible aux règles phonologiques, la TSR s'avère un modèle plus rigoureux et doté d'un un plus grand pouvoir de prédiction que la TSC. Harris (1996, p. 552) déclare cependant que tout en exprimant de manière appropriée l'idée de redondance des traits, les Théories de la sousspécification le font au prix d'un compromis sérieux avec l'identité phonétique des segments : les segments non spécifiés ne peuvent pas être prononcés ou entendus directement avant qu'ils soient convertis en segments pleinement spécifiés. Cette

3 La règle (11a) énonce qu'un segment qui n'est pas explicitement spécifié sur le trait de nasalité est par définition [- nasal]. Quant à la règle (11b), elle stipule que si le point d'articulation n'est pas indiqué, [coronal] est le trait de lieu par défaut. 
affirmation met l'accent sur deux points fondamentaux: premièrement, elle suggère que la phonologie doit renoncer à l'approche dérivationnelle transformant des segments faiblement spécifiés au niveau phonologique en segments surspécifiés au niveau phonétique, ce qui implique un abandon de la binarité à tous les niveaux d'analyse; deuxièmement, elle donne une nouvelle orientation à la phonologie vers la recherche de spécifications phonémiques susceptibles d'être entendues et prononcées à l'état isolé, telles que les primitives conçues par les TE.

Pour Kaye, Lowenstamm et Vergnaud $(1988$, p. 110) «[1]'unité première qui constitue le segment est l'élément, lequel est une matrice de traits entièrement spécifiée, phonétiquement interprétable». Chaque segment est composé soit d'un seul élément, soit d'une combinaison d'éléments dont chacun est «autonome» et «prononçable indépendamment». Voilà comment se présente selon eux le contenu des trois éléments principaux structurant les systèmes vocaliques:

$$
I=\left(\begin{array}{l}
\text { - arrondi } \\
- \text { arrière } \\
+ \text { haut } \\
- \text { ARL } \\
- \text { bas }
\end{array}\right)
$$$$
\mathrm{U}=\left(\begin{array}{l}
+ \text { arrondi } \\
+ \text { arrière } \\
+ \text { haut } \\
- \text { ARL } \\
- \text { bas }
\end{array}\right)
$$$$
\mathrm{A}=\left(\begin{array}{l}
- \text { arrondi } \\
+ \text { arière } \\
- \text { haut } \\
- \text { ARL } \\
+ \text { bas }
\end{array}\right)
$$

Les éléments $|\mathrm{I}|,|\mathrm{U}|$ et $|\mathrm{A}|$ correspondent aux trois voyelles les plus répandues typologiquement (Maddieson 1984, p. 125) et expriment respectivement les qualités suivantes: palatalité (ou acuité), arrondissement (ou gravité) et ouverture (ou compacité) (Schane 1984, p. 131). Ce sont également les voyelles dont l'acquisition est la plus précoce et qui sont les plus résistantes à la dégradation chez les aphasiques (Jakobson 1963, p. 136-142).

(13a)

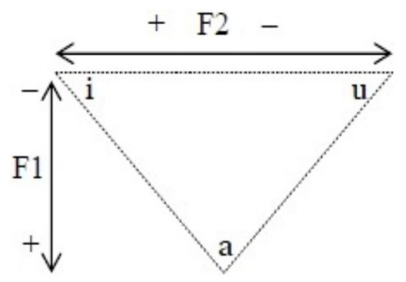

(13b)

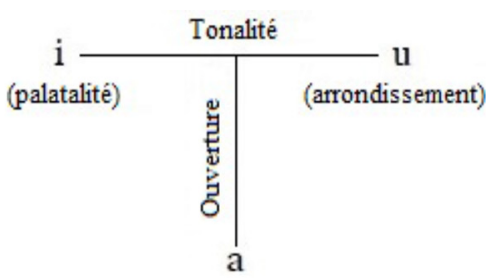

Stevens (1989) affirme que la haute perceptivité de /i, a, u/s'expliquerait par leur «effet quantal» ${ }^{4}$ dû à la présence de marques spectrales provoquées par la convergence de formants : de F2 et F1 chez/a/, de F2, F3 et F4 chez/i/, et de F1 et F0 chez /u/. En outre, il est bien connu que /a/ est différencié au maximum par rapport à /i/ et /u/ de par ses fréquences de $\mathrm{F} 1$, alors que /i/ et /u/ sont différenciés à

4 Selon l'auteur, les sons à effet quantal se distinguent par une grande saillance perceptive, dans la mesure où leur effet acoustique pourrait être atteint même en cas de légère variation de leur lieu d'articulation. 
l'extrême de par leurs fréquences de F2 (13a). En revanche, les voyelles d'avant non hautes /e, $\varepsilon /$, les voyelles hautes labialisées $/ y, u /$, les voyelles centrales $/ \mathfrak{i}$, o/, etc. se caractérisent par des fréquences de F2 et/ou de F1 ramenées à la moyenne, ce qui rend moins proéminents leurs contrastes avec la voyelle la plus proche de l'extrême périphérie du triangle vocalique. Cela étant, les systèmes vocaliques à trois termes, qui se ramènent presque toujours à la configuration $/ \mathrm{i}, \mathrm{a}, \mathrm{u} /$, peuvent être considérés comme une manifestation du principe de la dispersion optimale des voyelles dans l'espace acoustique (voir Liljencrants et Lindblom 1972, Lindblom 1986, Flemming 2004).

Quand il se réalise à l'état isolé, chacun des éléments $|\mathrm{I}|,|\mathrm{U}|$ et $|\mathrm{A}|$ s'identifie avec la voyelle correspondante des angles du triangle vocalique. Quand ils se combinent, en revanche, les trois éléments forment des voyelles composées à la manière des atomes qui s'assemblent pour la constitution de molécules, pour reprendre une métaphore courante en TE: si /e/, par exemple, peut s'interpréter comme un mélange de $|\mathrm{I}|$ et $|\mathrm{A}|$, /y/ serait le résultat de la fusion de $|\mathrm{I}|$ et $|\mathrm{U}|$, alors que /ø/ procéderait de l'assemblage de l'ensemble des trois éléments. Cette idée reflète de manière naturelle la notion de marque sans recourir à des conventions de marquage à la SPE. Il suffit d'admettre que le nombre des éléments contenus dans une voyelle est directement proportionnel à sa complexité :

$$
\begin{gathered}
/ \mathrm{i} /=/ \mathrm{I} / \\
/ \mathrm{y} /=/ \mathrm{I}+\mathrm{U} / \\
/ \varnothing /=/ \mathrm{I}+\mathrm{U}+\mathrm{A} /
\end{gathered}
$$

Mais puisque la simple opération de fusion en (14) ne suffirait pas pour capter toute la diversité des articulations vocaliques dans les langues, on admet l'existence de rapports hiérarchiques entres les éléments constitutifs du segment : par exemple, de gouvernement ou de dépendance. Van der Hulst (1993, p. 265) note à ce sujet qu'en Phonologie de dépendance la combinaison de $|\mathrm{I}|$ et $|\mathrm{A}|$ peut s'analyser aussi bien comme /e/ que comme /æ/, selon que c'est au premier ou au second élément qu'est dévolue la fonction de tête. L'élément-tête détermine le prototype de la voyelle composée, alors que le dépendant (ou opérateur) n'est responsable que du degré d'écart du prototype :
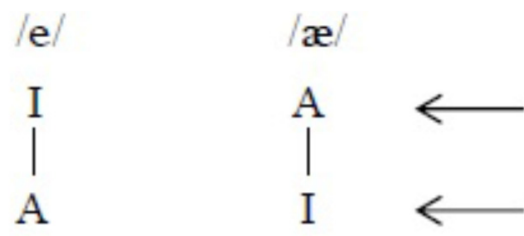

tête

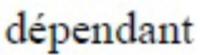


Pour empêcher que n'importe quels éléments se combinent avec n'importe quels autres, Kaye, Lowenstamm et Vergnaud (1988) introduisent la notion de charme, qui parachève l'allusion avec la chimie, tout en représentant un compromis sérieux avec les bases phonétiques de la phonologie. Ils admettent notamment que les éléments peuvent être «charmés» positivement ou négativement (en fonction de la qualité qu'ils expriment): ceux de même charme (par exemple, ++ ) se repoussent, alors que ceux de charme contraire (par exemple, +- ) s'attirent. Les auteurs précisent toutefois que le corrélat phonétique du charme serait la vocalicité, dans la mesure où celle-ci s'associe avec la notion de cavité de résonance, mais la vocalicité est plutôt une abstraction phonologique dont les corrélats phonétiques sont loin d'être univoques (voir Dogil 1993). En effet, à chacune des trois cavités supraglottiques est rattaché un élément de charme positif (16a); quant aux éléments censés être dépourvus de résonateur propre (et s'en procurer un en vertu de leur association avec d'autres éléments), ils sont considérés de charme négatif (16b).

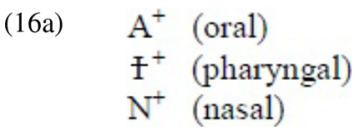

$$
\begin{array}{ll}
\mathrm{A}^{+} & \text {(oral) } \\
\mathrm{f}^{+} & \text {(pharyn } \\
\mathrm{N}^{+} & \text {(nasal) }
\end{array}
$$

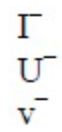

En cas de combinaison entre l'élément oral et l'élément pharyngal, ou entre l'élément pharyngal et l'élément nasal, ce mécanisme de blocage donne le résultat souhaité. Premièrement, les voyelles basses présentent une constriction nette au niveau du pharynx, alors que le mouvement de la racine linguale en avant provoque son élargissement. Deuxièmement, les voyelles nasales tendues sont typologiquement marquées parce que la nasalisation provoque une hausse des valeurs du F1, un élargissement de sa bande de fréquences, une diminution de son intensité et un affaiblissement de l'énergie totale de la voyelle, ces marques spectrales s'interprétant souvent sur le plan perceptif comme le résultat d'un abaissement du dos de la langue ou d'une centralisation dans l'espace acoustique (Ohala 1975, p. 294, Sampson 1999, p. 8). En outre, le lieu d'articulation d'une consonne nasale ne pourrait se trouver au-delà de la zone uvulaire puisqu'une constriction au niveau du pharynx ou de la glotte serait sans effet sur le couplage entre les cavités buccale et nasale (Ohala 1975, p. 294-295).

Charrette et Göksel (1994, p. 31) remarquent cependant que la notion de charme, censée bloquer la combinaison de $\left|\mathrm{A}^{+}\right|$avec $\left|\mathrm{N}^{+}\right|$, fait incorrectement des voyelles nasales basses des segments marqués. Il est bien connu en effet que la nasalisation d'une voyelle est d'autant plus facile que celle-ci est ouverte parce que l'abaissement du dos de langue est normalement accompagné d'un abaissement automatique du voile du palais (Ohala 1975, p.299, 
Ladefoged 1989, p. 56) ${ }^{5}$. Cela conduit Charrette et Göksel $(1994$, p. 32) à abandonner l'élément $|\mathrm{H}|$ (sous proposition de Jonathan Kaye), de même que le charme comme mécanisme phonologique, et à distinguer les voyelles tendues (ou [ + ARL]) des voyelles non tendues (ou [- ARL]), en posant que les premières posséderaient une tête réelle, alors que les secondes seraient des expressions à tête nulle (symbolisée ci-dessous par _):

$$
\begin{aligned}
& \mathrm{e}=(\mathrm{A} \cdot \mathrm{I}) \quad \mathrm{o}=(\mathrm{A} \cdot \mathrm{U}) \\
& \varepsilon=\left(\mathrm{A} \cdot \mathrm{I} \cdot \_\right) \quad \mathrm{o}=\left(\mathrm{A} \cdot \mathrm{U} \cdot \_\right)
\end{aligned}
$$

Or, cette solution rend la théorie encore plus abstraite, tout en affaiblissant son pouvoir explicatif. À la question «Qu'est-ce qui empêche [alors] certains éléments de se combiner, si ce n'est pas la valeur de leur charme?» l'article répond tout simplement: «rien» (p.35). Entre le formalisme plus rigoureux, basé sur un nombre plus élevé d'éléments et de restrictions combinatoires, et la réduction des primitives (éléments et charme) au risque que cela engendre des segments impossibles, les deux auteurs choisissent la seconde alternative. Cela revient à admettre que la fusion d'éléments peut être autorisée ou bloquée en fonction de conventions spécifiques pour chaque langue, lesquelles ne sont pas très différentes de par leur mode d'énonciation de celles dont font usage les théories subsegmentales standard.

Un autre procédé courant pour restreindre la combinatoire des éléments en TE est la fusion de lignes autosegmentales. Polgárdi (1998, p. 151-152) note à ce sujet que dans les systèmes trivocaliques $/ \mathrm{i}, \mathrm{a}, \mathrm{u} /$, comme celui quechua, les trois éléments devraient être disposés sur une même ligne, ce qui les empêcherait de se combiner et de former des voyelles dérivées (18a). Dans un système pentavocalique /i, e, a, o, u/, comme celui de l'espagnol, en revanche, seul l'élément $|\mathrm{A}|$ occuperait une ligne autonome, puisqu'il devrait fusionner aussi bien avec $|\mathrm{I}|$ qu'avec $|\mathrm{U}|$. Quant aux deux derniers, ils partageraient une même ligne afin de ne pas s'assembler (18b).
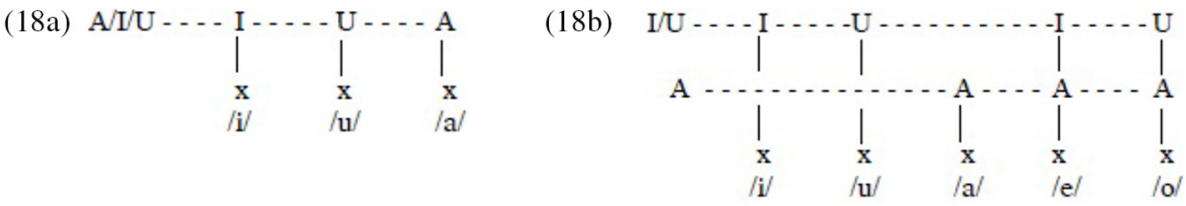

5 Cela s'explique par l'existence de liens musculaires entre la langue le vélum, ainsi que par l'action du muscle palatopharyngien, responsable à la fois de l'abaissement du vélum et du resserrement du pharynx (Trigo 1991, p. 131). 
D'après l'auteure, le caractère marqué de (18b) par rapport à (18a) se traduit directement par l'élargissement des tires autosegmentales. Or, sur le plan typologique, cette position s'avère incorrecte, puisque c'est effectivement le système en (18b) qui est le plus diffusé dans les langues (Maddieson 1984, p. 136, Lindblom 1986, p. 16, 21), alors que celui en (18a) est relativement rare. Ainsi, la complexification de la structure élémentaire des voyelles n'implique pas nécessairement une complexification du système vocalique tout entier.

Il ne serait pas injustifié de dire que les principes de base des TE, conçus à l'origine pour la description des voyelles, se voient ébranlés après les tentatives de les appliquer également à celle des consonnes (voir notamment Kaye, Lowenstamm et Vergnaud 1990, Harris 1990, 1996, Scheer 1999). C'est effectivement l'étude de la structure élémentaire des consonnes qui conduit à augmenter le nombre des éléments, ainsi qu'à réviser leur contenu. Boltanski (1999, p. 85) note à ce sujet que si $|\mathrm{I}|,|\mathrm{A}|$ et $|\mathrm{U}|$ ont un contenu réel, dans la mesure où il est réellement possible de les entendre ou de les prononcer à l'état isolé, toutes les consonnes - y compris les moins marquées parmi elles (comme /t, k, p/) - sont conçues comme un mélange d'éléments. Qui plus est, des éléments comme $|\mathrm{H}|,|\mathrm{L}|^{6}$, $|\mathrm{f}|$, etc. sont inaccessibles par voix auditive ou articulatoire, à l'instar des éléments quantaux, ce qui leur réserve le statut d'une espèce de traits monovalents.

Alors que le binarisme rompt avec la conception atomique du segment comme indécomposable en unités plus petites, les TE opèrent une sorte de «réatomisation » de ses particules constitutives. Celles-ci sont considérées comme une espèce d'éléments-segments pouvant exister aussi bien à l'état isolé qu'à l'état moléculaire (en fusion avec d'autres éléments). À propos de cette métaphore courante, Boltanski (1999, p. 87) explique :

[J]e ne crois pas vraiment que KLV croient vraiment que les particules sont des objets de la nature comme les atomes. Elles existent dans un univers mental (conditionné ou corrélé biologiquement), mais cet univers mental est étranger à ce que nous croyons savoir, à nos processus conceptuels ordinaires, à nos intuitions préthéoriques [...]. Bref, il est comme une nature, a priori inconnue et imprévisible, à laquelle il faut "arracher ses secrets".

Ces mots sont une critique du formalisme abstrait, porté à son paroxysme par les TE, qui renforce l'hermétisation de la phonologie entamée par les générativistes. Désormais, les éléments et les structures que manipule la phonologie ne sont plus accessibles (uniquement) par une connaissance de la réalité phonétique qu'elle

$6|\mathrm{H}|=$ cordes vocales tendues/absence de ton fondamental, $|\mathrm{L}|=$ cordes vocales relâchées/ voisement. 
devrait refléter, analyser et schématiser, mais (aussi) par une connaissance de ses propres lois internes qui ne sont pas nécessairement celles en vigueur dans le monde physique réel.

Pour employer une métaphore un peu approximative, si la GT (avec ses nœuds, racines et arborescences) pourrait s'identifier avec une sorte de mouvance impressionniste en phonologie segmentale, les TE, elles, devraient correspondre à sa période surréaliste. Dans cet ordre d'idées, la Phonologie articulatoire (PA) (Browman et Goldstein 1986, 1987, 1989, 1992) est plutôt une tentative de retourner au réalisme phonétique. Pour ses fondateurs, elle est une réaction contre "le fossé entre la structure linguistique et la structure physique de la parole», creusé par les cadres adoptant "des spécifications statiques» pour la description des changements phonologiques. D'après eux, ce fossé ne peut être comblé que par un modèle incorporant l'idée de mouvement (Browman et Goldstein 1986, p. 219, 224).

\subsection{Les gestes de la Phonologie articulatoire}

La PA pose que l'élément phonologique élémentaire, indissociable en entités plus petites, est le geste, qui est une unité abstraite, discrète et dynamique, possédant une nature spatio-temporelle (Browman et Goldstein, 1987, p. 2). Le caractère discret et abstrait des gestes est dû à ce qu'ils connaissent, en tant que corrélats phonologiques de mouvements articulatoires, une existence autonome et indépendante du contexte phonétique: par leur présence ou absence, ainsi que par leurs paramètres dynamiques ou leur synchronisation les uns par rapport aux autres dans les représentations phonologiques, ils assurent le contraste entre les items lexicaux. Quant à leur nature dynamique, elle découle de ce qu'ils reflètent des événements physiques réels dans le temps et dans l'espace, consistant à former ou à relâcher des constrictions dans le conduit vocal. Puisque les gestes ont «leur propre durée» et représentent «des événements physiques», ils ont la propriété «de se superposer les uns aux autres» et d'être "affectés par des processus physiques dans l'acte de la parole» (1992, p. 26). Enfin, en tant que primitives, les gestes s'organisent en "constellations» pour la constitution des phonèmes, métaphore qui revient dans les quatre articles de Browman et Goldstein cités ici.

Les auteurs précisent que le geste diffère à la fois du trait et du segment. D'une part, il est une unité phonologique supérieure au trait, dans la mesure où il reflète un mouvement articulatoire à caractère distinctif, déterminé par un ensemble de paramètres: choix d'articulateurs actifs, localisation et magnitude de la constriction, etc. D'autre part, il est plus petit que le segment, parce qu'il est parfois nécessaire que plusieurs gestes soient coordonnés pour la formation d'une unité segmentale (1989, p. 210-211). 
En PA, l'organisation et la coordination des gestes dans l'espace-temps sont représentées sous la forme d'une partition gestuelle (angl. gestural score), illustrée par Browman et Goldstein (1992, p. 28) avec le mot anglais palm $\left[\mathrm{p}^{\mathrm{h}} \mathrm{a}: \mathrm{m}\right]$ :

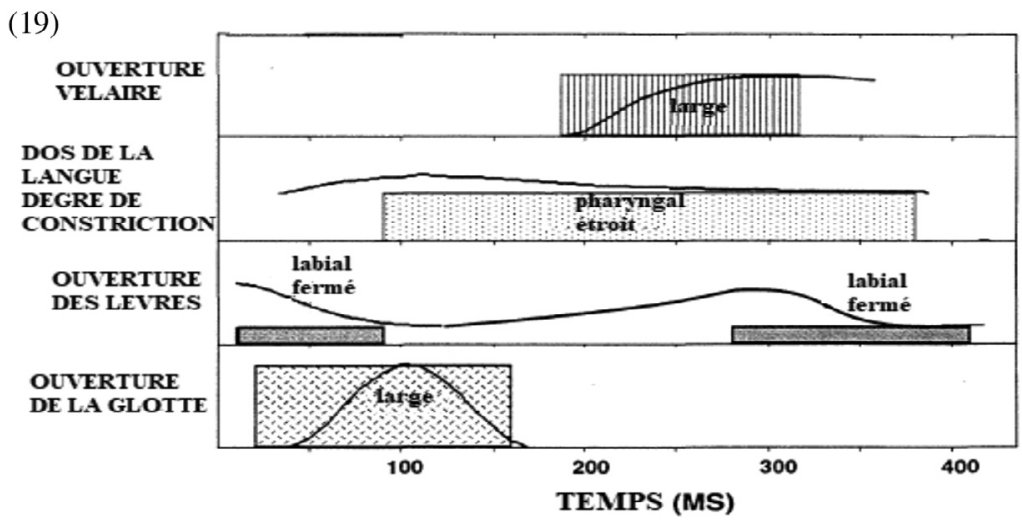

Les gestes reflétant l'ouverture de la cavité nasale (dans $/ \mathrm{m} /)$, la constriction du pharynx (dans /a/), l'occlusion formée par les lèvres (dans $/ \mathrm{p} /$ et $/ \mathrm{m} /$ ), et l'élargissement de la glotte (pendant l'aspiration), sont représentés sous la forme de rectangles disposés sur quatre paliers. À chaque palier, la magnitude de la constriction est exprimée aussi bien par la hauteur des rectangles, que par des courbes indiquant les modifications dans la configuration du tractus vocal. Plus le point atteint est élevé, plus la constriction sera large.

D'autre part, les gestes en (19) sont coordonnés les uns par rapport aux autres également sur l'axe temporel. La longueur des rectangles correspond au laps de temps pendant lequel la variable en question est activée. Ainsi, la fermeture labiale occupe-t-elle approximativement l'intervalle entre 10 et $90 \mathrm{~ms}$. En même temps, à la $20^{\mathrm{e}} \mathrm{ms}$ commence l'élargissement de la glotte, responsable de l'aspiration de la plosive. Le geste le plus prolongé de la partition est le resserrement du pharynx, correspondant à la voyelle et empiétant sur tous les autres gestes. Quant à l'abaissement du vélum, il anticipe sur la seconde fermeture labiale (de quelques $100 \mathrm{~ms}$ ), ce qui provoque une nasalisation de $/ \mathrm{a} /$.

La PA est parfaitement adaptée pour capter des modifications dans le timing des gestes à la suite d'une accélération du débit d'élocution. Cela est illustré par les deux auteurs avec le syntagme perfect memory prononcé lors d'un débit normal (20a) et d'un débit rapide (20b). Dans le premier cas, la pause entre les mots permet d'entendre clairement l'explosion du/t/ final, alors que dans le second cas, l'occlusion alvéolaire se voit complètement obscurcie par l'occlusion labiale qui 
vient s'y superposer. L'effet perceptif de ce «masquage gestuel» (angl. gestural hiding) est la chute apparente de /t/, dont les marques de lieu (comme l'emplacement du bruit d'explosion sur le spectre) ne peuvent pas être identifiés.

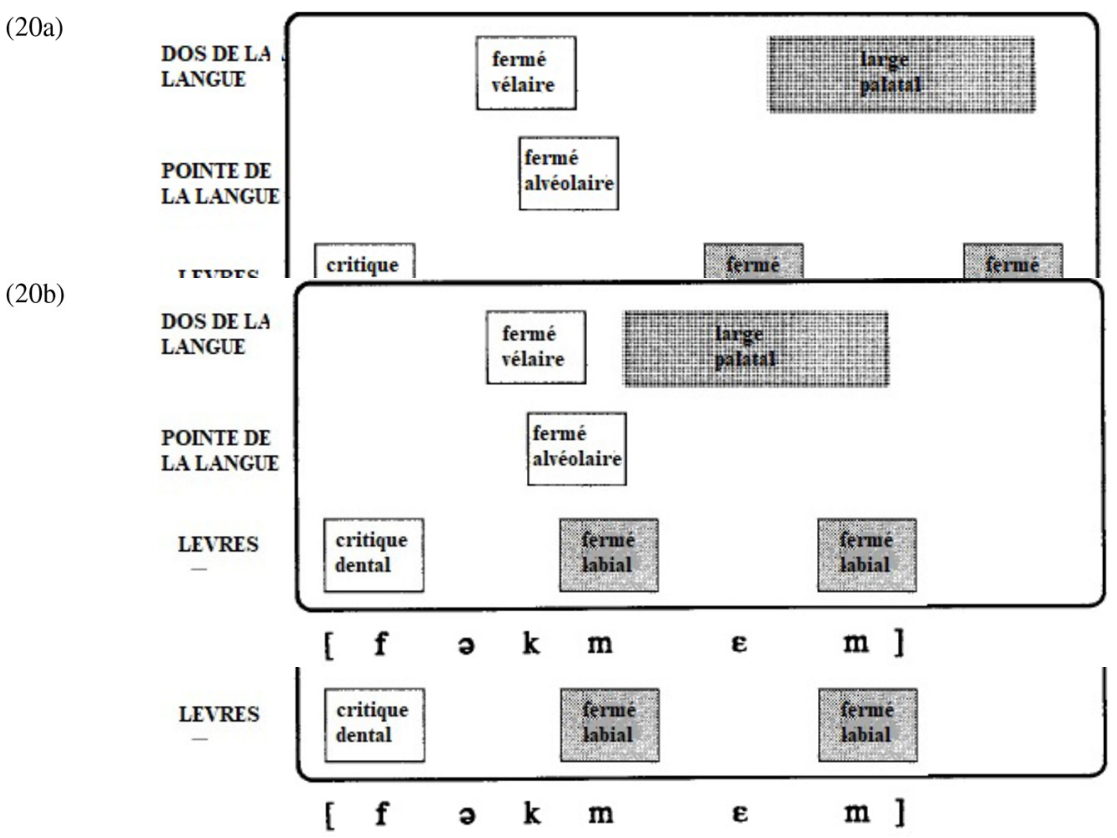

Des données cinéradiographiques citées par Browman et Goldstein (1987, p. 20) montrent en effet une articulation coronale nettement présente à la fin du mot perfect même en cas de débit rapide, ce qui prouve que la chute de /t/ n'est qu'un effet auditif des mouvements articulatoires qui s'enchevêtrent. Selon les auteurs, ces faits démontreraient la supériorité du formalisme de la PA par rapport à celui de la GT, dans la mesure où leur modèle n'a pas à recourir à des opérations de suppression de segments qui «s'effacent». Ils affirment à ce propos qu'aucun geste ne peut être supprimé, ajouté ou transformé en un autre geste, la variation articulatoire découlant soit de la superposition des gestes, soit de leur réduction spatio-temporelle.

Même si la PA est née d'un désir de lier plus étroitement la phonologie à la phonétique, certains de ses opposants lui reprochent précisément de rester trop près des structures superficielles, et notamment des mécanismes de production de la parole, pour être un vrai modèle phonologique. Faisant la synthèse des critiques qui lui ont été adressées, Fougeron (2005, p. 287) signale que la PA ne peut capter de manière adéquate ni l'énorme diversité des sons attestés dans les langues du monde, ni la spécificité des processus post-lexicaux, ni la notion de classe naturelle. 
D'après nous, l'inconvénient majeur de la PA est que, en tant que modèle à orientation phonétique, elle néglige les corrélats acoustiques des gestes ou les considère tout au plus comme un simple effet des mouvements articulatoires. Or, certains changements sont déclenchés par la confusion de segments perceptivement similaires mais articulés de manière très différente (voir Ohala et Lorentz 1977) et leur motivation ne saurait donc être captée par un modèle de ce genre.

Pour ce qui est du reproche que la PA ne fait que visualiser schématiquement la dynamique des articulateurs sans formuler des contraintes explicites pour limiter la surpuissance générative de son formalisme, ce n'est pas nécessairement un défaut. La question si et comment la phonologie doit modéliser la substance phonique sera discutée brièvement dans la section suivante.

\section{CRitiques du Formalisme abSTRAit}

Il est bien connu que le CLP contribue fortement à l'émancipation de la phonologie, conçue comme une science humaine, relativement à la phonétique, considérée comme rattachée aux sciences naturelles. En témoigne cette thèse de Troubetzkoy (1986, p.3), qui inscrit les deux disciplines dans la dichotomie saussurienne langue/parole : «la science des sons de la parole, ayant affaire à des phénomènes physiques concrets, doit employer les méthodes des sciences naturelles; la science des sons de la langue doit au contraire employer des méthodes purement linguistiques ». Même si, plus tard, Jakobson et ses collaborateurs, suivis de Chomsky et Halle, ont cherché à trouver pour les traits distinctifs des corrélats acoustiques ou articulatoires, leur binarisme est contreintuitif pour la phonétique dont la matière n'est pas discrète, et encore moins bipolaire. Toutes les tentatives ultérieures de défendre, restreindre, rejeter ou remplacer la binarité des primitives ont donné lieu à un foisonnement terminologique et à un formalisme de plus un plus abstraits. Ainsi, l'émancipation et l'autonomisation de la phonologie ont-elles conduit finalement à son hermétisation.

Liljencrants et Lindblom (1972) et Ohala $(1983,1985)$ mettent ceux qui étudient les universaux dans les comportements des segments et dans les systèmes segmentaux au défi de porter un regard sur ce qui se produit réellement dans le monde physique, physiologique, psychologique et social. Selon Ohala (1985, p. 228), dans tous ces domaines, on peut trouver un certain nombre de primitives susceptibles d'expliquer les patrons sonores des langues. Ohala (1983, p. 190) affirme notamment que des processus a priori aussi divers que la variation allophonique, le changement phonologique, la variation dialectale, l'alternance morpho-phonémique et la structuration des inventaires segmentaux, peuvent être conçus comme « des manifestations d'un seul et même phénomène pris à des stades 
différents ou vu sous des angles différents ». Ainsi, la variation allophonique est le résultat de l'anatomie spécifique du tractus vocal mais peut donner lieu à un changement catégoriel en cas de phonologisation des allophones. Le changement phonologique, de son côté, peut se retrouver à l'origine d'une variation dialectale, s'il n'affecte que le parler d'une seule communauté, ou d'une alternance morphophonémique, s'il n'affecte qu'un seul morphème dans un contexte segmental précis. Enfin, une conséquence possible du changement phonologique est l'apparition d'un nouveau segment dans un inventaire phonémique.

Anti-formaliste fervent, Ohala se déclare pour l'approche phonétique intégrée en phonologie, marquée par le soin d'y inclure des explications relevant des domaines de l'anatomie du conduit vocal, de l'aérodynamique, de l'acoustique et de la perception de la parole. Même s'il faut réduire au minimum les éléments primaires de toute théorie, afin qu'ils ne soient redondants les uns par rapport aux autres, d'après lui, verser dans des modèles réductionnistes (comme les TE, par exemple), "pourrait retarder la phonologie de la même façon que la chimie occidentale fut retardée par la théorie prématurée selon laquelle les composantes primaires de la matière seraient la terre, l'air, l'eau et le feu » (Ohala 1985, p. 228) ${ }^{7}$.

Ohala (1992, p. 180) s'oppose à ce que des «notions abstraites» comme le Principe du contour obligatoire, la Contrainte de non-croisement des lignes ou d'inaltérabilité des géminées (en phonologie autosegmentale) soient prises pour des «éléments réels». Contrairement à la loi de Boyle-Mariotte, par exemple, qui explique non seulement le dévoisement des plosives, mais aussi le fonctionnement de nombreux objets de notre quotidien (pompes à bicyclette, ballons, baromètres), les conventions abstraites ne semblent en effet applicables qu'en phonologie (Ohala 1990, p. 267). Celles-ci sont pour lui des «primitives formelles» dont le pouvoir explicatif ne découle pas de la substance phonique: "prises isolément, elles sont comme les ingrédients d'un gâteau - farine, sucre, œufs, etc. - lesquels, même placés bout-à-bout sur le comptoir de cuisine, ne se transformeront jamais en gâteau. On doit savoir aussi comment mélanger ces choses-là ${ }^{8}$ (Ohala 1985, p. 228).

Ohala et Kawasaki (1984, p. 113), ironisant sur le formalisme des théories phonologiques, ont recours à une autre amusante métaphore culinaire. Ils comparent ces théories à un restaurant très «high-tech » où il suffit d'appuyer sur des boutons pour commander son repas. Une fois que le client a commandé le menu désiré au moyen du clavier, son plat lui parvient automatiquement grâce à un tapis roulant sortant de la cuisine. Il ne peut pas voir ce qui se passe dans la cuisine, ni demander à un serveur comment on prépare les plats. La spécialité du restaurant est 
un sandwich ouvert avec du pain, du beurre de cacahuètes et de la mayonnaise. Or, lorsqu'un client n'appuie pas sur les boutons dans l'ordre correct pour commander cette prétendue spécialité, rien ne sort de la cuisine. Selon les auteurs, il y aurait deux approches pour voir où est le problème. La première est l'approche formelle qui consiste à inventer une grammaire qui n'engendre que des sandwichs possibles; il est essentiel dans ce cas de spécifier l'ordre correct d'application des opérations successives : il faut commander d'abord le pain et ensuite seulement le beurre de cacahuètes et la mayonnaise, mais on ne peut pas commander de la mayonnaise sans avoir commandé du beurre auparavant. La deuxième est l'approche substantielle qui consiste à chercher le problème dans la nature même des ingrédients : lequel d'entre eux est plus gluant, plus lisse et compatible avec les autres ou se déforme plus facilement.

Le tapis roulant, le clavier et l'empilement vertical des ingrédients représentent sans doute le dérivationnisme, le générativisme et les représentations multilinéaires des théories phonologiques passées en revue. Pour rester dans l'imagerie créée par Ohala, beaucoup de ces théories suivent fidèlement la «bonne recette» pour «les sandwiches possibles » non seulement en s'en tenant à leur propre formalisme mais aussi à celui de la Théorie de l'optimalité (TO) (Prince et Smolensky 1993, McCarthy et Prince 1993): c'est à celle-ci que revient parfois le choix de la représentation optimale parmi plusieurs où l'on peut voir une propagation autosegmentale de traits (Parkinson 1996, Ringen et Heinämäki 1999), une combinaison d'éléments (Polgárdi 1998) ou de gestes articulatoires (Jun 1995). De cette manière, on choisit l'approche formelle pour la production du «sandwich souhaité » (comme métaphore de la parole), indépendamment de la nature des ingrédients primaires (traits, éléments ou gestes). Il suffit seulement d'introduire un certain nombre de contraintes hiérarchisées pour prévenir les effets secondaires du processus de production (c'est-à-dire pour faire en sorte que le mécanisme d'évaluation de la TO élimine les candidats indésirables).

Prenons à titre d'exemple la contrainte NoCoda (Prince et Smolensky, 1993, p. 34) qui exprime l'interdiction suivante: «Les syllabes ne doivent pas posséder de coda $»$. Cette affirmation reflète l'observation que toutes les langues possèdent des syllabes ouvertes, mais pas nécessairement des syllabes fermées, ainsi que la tendance universelle à syllaber les consonnes intervocaliques à droite et non à gauche. Cela rend le format CV le seul type syllabique universellement attesté (Jakobson 1963, p. 119, 136, Clements et Keyser 1983, p.29), d'où une autre contrainte énonçant que «les syllabes doivent posséder une attaque » (Prince et Smolensky, 1993, p.93). Ces contraintes ne signalent cependant qu'un fait translinguistique sans chercher à comprendre sa motivation. Scheer (1999) déclare à ce sujet que les faits reflétés par une contrainte ne sont presque jamais considérés comme découlant d'un principe général et indépendant de l'observation. En outre, 
les contraintes, d'après lui, induisent un raisonnement circulaire, poussant intuitivement ceux qui en font usage à cesser de se poser davantage de questions et de poursuivre les recherches: "si la réponse à la question POURQUOI est la contrainte elle-même, il est inutile de chercher une explication des faits » (p. 202203). Pour illustrer cela, il évoque la découverte de la loi de la gravitation: lorsque les pommes se détachent d'un arbre, elles ne montent pas vers le haut, ni restent en l'air, mais tombent verticalement. Si ce comportement avait été mis par les physiciens sur le compte de la contrainte «Les pommes doivent tomber verticalement», on n'aurait probablement jamais découvert le lien entre les pommes tombantes, leur masse et la masse de la Terre. Grâce à la découverte que les masses s'attirent, il devient possible d'appliquer la loi de la gravitation à tous les objets possédant une masse et de prévoir ainsi leur comportement possible et impossible.

L'approche substantielle évoquée par Ohala et Kawasaki consisterait dans le cas des contraintes syllabiques de la TO à chercher plutôt les corrélats physiques et physiologiques de la régularité qu'elles expriment, afin de voir pourquoi elle est universelle. Les deux auteurs notent à ce sujet que le centre perceptif de la syllabe, responsable de son identification auditive, se trouve près de ses transitions $\mathrm{CV}$, c'est-à-dire à l'intersection entre l'attaque et le noyau. Cela peut être mis sur le compte du fait que c'est précisément dans ce tronçon de la syllabe que les modulations acoustiques (modifications au niveau de l'intensité, de la périodicité, $\mathrm{du}$ ton fondamental, du spectre) sont les plus saillantes. Ces modulations, de leur côté, sont attribuables à ce que les obstruantes (qui sont en principe plus nombreuses que les sonantes dans les langues du monde) tendent à provoquer, lors du relâchement de leur constriction, des modifications dans les paramètres aérodynamiques de la pression et du flux d'air, qui sont plus abruptes qu'au moment de l'établissement de la constriction. Par ailleurs, comme la coarticulation est plutôt régressive que progressive et comme les articulations vocaliques subissent davantage de modifications acoustiques sous l'effet des articulations consonantiques que l'inverse, le spectre d'une voyelle dans une séquence $\mathrm{C}_{1} \mathrm{VC}_{2}$ sera affecté davantage par $\mathrm{C}_{2}$ que par $\mathrm{C}_{1}$, ce qui revient à dire qu'il aura plus d'affinité avec celui de la consonne de coda qu'avec celui de la consonne d'attaque. Et comme le contraste perceptif est directement proportionnel à l'importance des changements dans les paramètres acoustiques, on peut conclure que les séquences $\mathrm{CV}$ sont perceptivement plus proéminentes que les séquences VC. Ce ne sont que quelques-unes des raisons possibles pour que les segments et les oppositions entre eux soient synchroniquement mieux représentés et diachroniquement plus stables en position d'attaque qu'en position de coda. Pour un tableau plus détaillé des faits, le lecteur intéressé pourra consulter l'article en question, et plus concrètement les pages 115-119. 
Si l'on retourne maintenant à l'apologue du restaurant, on peut y trouver deux autres métaphores : la cuisine où personne ne peut voir ce qui se passe évoque sans ambiguïté la réalité mentale à laquelle les phonologues dès la parution de SPE essaient d'accéder par les règles de transformation de structures profondes en structures de surface; quant au personnel manquant et à l'impossibilité de se renseigner sur le mode de préparation des plats, c'est sans doute une critique de l'absence dans de nombreux travaux de phonologie de suffisamment de données expérimentales obtenues à la suite d'enquêtes avec des informateurs.

À ce sujet, parmi les postulats de base de la Phonologie de laboratoire (Pierrehumbert, Beckman et Ladd 2000, D'Imperio 2005), on trouve la thèse que les comportements des systèmes phonologiques peuvent être explorés avec les méthodes des "mature sciences", comme la physique et la biologie, et que le progrès de la théorie phonologique n'est possible qu'à condition de tester expérimentalement le pouvoir de prédiction des modèles. D'Imperio (2005, p. 244) est d'avis que la modélisation est une partie importante et inséparable de la linguistique théorique. Elle est même obligatoire lors du développement d'applications ou en synthèse/reconnaissance de la parole où il s'agit de recourir à des modèles dont il faut tester mathématiquement et expérimentalement la validité, l'opérationnalité et la calculabilité.

\section{EN GUISE DE CONCLUSION}

Nous avons déjà soutenu que la formalisation est nécessaire non en tant que cadre théorique qui se suffirait à lui-même et serait doté d'un pouvoir de génération, d'explication ou de prédiction découlant de ses propres principes, mais en tant que moyen de schématisation et de visualisation de la dynamique des processus phonologiques (Burov 2013, 2017). Ainsi, quoique représentant une opération abstraite, la propagation autosegmentale illustre de façon réussie et simple au moins deux idées fondamentales : la durée accrue de l'émission du trait propagé et l'enchevêtrement des mouvements articulatoires se réalisant simultanément dans le cadre de plusieurs segments. En d'autres termes, lorsqu'on recourt à des opérations et à des primitives abstraites, il est important de chercher leurs manifestations et corrélats phonétiques concrets; ou plus exactement, quand on analyse des événements relevant de la substance sonore du langage, il convient de les illustrer par des représentations phonologiques appropriées à leur dynamique et à leur motivation.

La phonologie ne doit pas se contenter d'expliquer le déclenchement ou le blocage des processus segmentaux par des contraintes ou des conventions formelles. Désireuse de formaliser les notions et les phénomènes phonologiques de façon simple et élégante, elle finit souvent par s'enfermer dans ses propres 
représentations et se détacher de la matière sonore, proposant parfois des solutions qui ne correspondent à rien au niveau de l'audition ou de la production. Or, les représentations phonologiques ne sont pas une réalité en soi; elles ne sont réelles que dans la mesure où elles reflètent les propriétés de la substance physique de la langue.

\section{BIBLIOGRAPHIE}

Anderson, John et Ewen, Colin, 1987. Principles of Dependency phonology, Cambridge, Cambridge University Press.

Archangeli, Diana, 1988. «Aspects of Underspecification theory», Phonology 5, 183-207. Archangeli, Diana et Pulleyblank, Duglas, 1994. Grounded phonology, Cambridge, MIT Press.

Avery, Peter et Rice, Keren, 1989. «Segment structure and coronal underspecification », Phonology 6, 179-200.

Bloomfield, Leonard, 1973. Language, London, George Allen and Unwin ( $1^{\text {st }}$ edition, 1933).

Boltanski, Jean-Elie, 1999. Nouvelles directions en phonologie, Paris, PUF.

Browman, Catherine et Goldstein, Louis, 1986. "Towards an Articulatory phonology», Phonology Yearbook 3, 219-252.

- 1987. "Tiers in Articulatory phonology, with some implications for casual speech, Haskins Laboratories Status Report on Speech Research, SR-92, 1-30.

- 1989. "Articulatory gestures as phonological units», Phonology, vol. 6, n 2, 201-251.

- 1992. «Articulatory phonology: an overview», Phonetica 49, 155-180.

Burov, Ivaylo, 2013. Les phénomènes de sandhi dans l'espace gallo-roman, Sofia, Presses universitaires St. Clément d'Ohrid.

- 2017. Фонетични и фонологични универсалии при асимилационните процеси. Теория за относителната бинарност и йерархия на признаците (trad. Universaux phonétiques et phonologiques dans les processus d'assimilation. Théorie de la binarité et de la hiérarchie relatives des traits), Sofia, CU Romanistika.

Carvalho, Joaquim Brandão de, 1993. "De quoi sont faites les voyelles? Phonologie tridimensionnelle des particules et harmonie vocalique », Laks, Bernard et Plénat, Marc (éd.), De natura sonorum. Essais de phonologie, Paris, Presses universitaires de Vincennes, 65-100.

Charrette, Monik et Göksel, Asli, 1994. «Switching and vowel harmony in Turkic languages », SOAS Working Papers in Linguistics and Phonetics 4, Lublin, University Press of the Catholic University of Lublin, 31-52.

Chomsky, Noam, 1957. «Syntactic structures», Janua linguarum 4, The Hague, Mouton.

Chomsky, Noam et Halle, Morris, 1968. The sound pattern of English, New York, Harper \& Row.

Clements, George, 1985. «The geometry of phonological features », Phonology Yearbook $2,225-252$.

- 1988. "Toward a substantive theory of feature specification», Papers from the Annual meeting of the North East Linguistic Society 18, 79-93.

- 1993a. "Un modèle hiérarchique de l'aperture vocalique : le cas bantou », Laks, Bernard et Plénat, Marc (éd.), De natura sonorum. Essais de phonologie, Paris, Presses universitaires de Vincennes, 23-64.

- 1993b. «Lieu d'articulation des consonnes et des voyelles », Laks, Bernard et Rialland, Annie (dir.), Architecture des représentations phonologiques, Paris, Éditions du CNRS, 101-145.

Clements, George et Hume, Elizabeth, 1995. « The internal organization of speech sounds », Goldsmith, John (ed.), Handbook of Phonological Theory, Oxford, Blackwell, 245-306. 
Clements, George et Keyser, Samuel, 1983. CV Phonology, a Generative theory of syllable. Cambridge, MIT Press.

Courtenay, Jan Baudouin de, 1895. Versuch einer Theorie phonetischer Alternationen; ein Capitel aus der Psychophonetik, Strasbourg, K. J. Trübner.

D'Imperio, Mariapaola, 2005. "Phonologie de laboratoire: notions de base et applications », Nguyen, Noël et al. (éds.), Phonologie et phonétique: forme et substance, Paris, Hermès, 240-264.

D’Intorno, Francesco et Weston, Rosemary, 2000. «Vowel alternation, vowel/consonant assimilation and OCP effects in a Barese dialect», Repetti, Lori (ed.), Phonological theory and the dialects of Italy, Amsterdam/Philadelphia, John Benjamins Publishing Co, 89-110.

Dogil, Grzegorz, 1993. "La phonologie peut-elle renoncer aux traits distinctifs de classe supérieure? », Laks, Bernard et Rialland, Annie (dir.), Architecture des représentations phonologiques, Paris, Editions du CNRS, 187-201.

Durand, Jacques, 1990. Generative and Non-Linear phonology, London, Longman.

- 2005a. «La phonétique classique: 1'Association phonétique internationale et son alphabet», Nguyen, Noël et al. (éd.), Phonologie et phonétique: forme et substance, Paris, Hermès, 25-59.

- 2005b. «Les primitives phonologiques: des traits distinctifs aux éléments », Nguyen, Noël et al. (éds.), Phonologie et phonétique : forme et substance, Paris, Hermès, 63-93.

Flemming, Edward, 2004. "Contrast and perceptual distinctiveness », Hayes, Bruce et al. (eds.), Phonetically based phonology, Cambridge University Press, 232-276.

Fougeron, Cécile, 2005. «La Phonologie articulatoire : une introduction », Nguyen, Noël et al. (éds.), Phonologie et phonétique: forme et substance, Paris, Hermès, 265-290.

Fromkin, Victoria, 1970. "The concept of 'naturalness' in universal phonetic theory », Glossa 4 (1), 29-45.

Gerlach, Sharon, 2010. The acquisition of consonant feature sequences: harmony, metathesis and deletion patterns in phonological development (Ph.D. dissertation), University of Minnesota.

Goldsmith, John, 1976. Autosegmental phonology, Ph. dissertation, MIT.

Halle, Morris, 1957. "In defense of the number Two», Pulgram, Ernst (ed.), Studies presented to Joshua Whatmough, The Hague, Mouton, 65-72.

- 1964. «Phonology in Generative grammar», Fodor, Jerry et Katz, Jerrold (eds.), The structure of language: readings in the philosophy of language, 334-352.

- 1995. «Feature geometry and feature spreading », Linguistic Inquiry, vol. 26, $\mathrm{n}^{\circ}$ 1, 1-46.

- 2005. «Palatalization/velar softening: what it is and what it tells us about the nature of language », Linguistic Inquiry 36/1, 23-41.

Halle, Morris, Vaux, Bert et Wolfe, Andrew, 2000. «On feature spreading and the representation of place of articulation », Linguistic Inquiry 31/3, 387-444.

Harris, John, 1990. "Segmental complexity and phonological government», Phonology Yearbook 7, 255-300.

- 1996. «Phonological output is redundancy-free and fully interpretable», UCL Working Papers in Linguistics 8, 551-574.

Hayes, Bruce, 1995. Metrical stress theory. Principles and cases studies, Chicago and London, University of Chicago Press.

Jakobson, Roman, 1963. Essais de linguistique générale, Paris, Les Éditions de Minuit.

Jakobson, Roman, Fant, Gunnar et Halle, Morris, 1952. Preliminaries to speech analysis, Cambridge, MIT Acoustics laboratory technical report 13.

Jakobson, Roman et Halle, Morris, 1956. Fundamentals of language, The Hague, Mouton. Jun, Jongho, 1995. Perceptual and articulatory factors in place assimilation: an Optimality theoretic approach, University of California, Los Angeles.

Kaye, Jonathan et Lowenstamm, Jean, 1984. « De la syllabicité », Dell, François et al. (éds.), Forme sonore du langage, Paris, Hermann, 123-159. 
Kaye, Jonathan, Lowenstamm, Jean et Vergnaud, Jean-Roger, 1988. « La structure interne des éléments phonologiques : une théorie du charme et du gouvernement », Recherches Linguistiques de Vincennes 17, 109-134.

- 1990. «Constituent structure and government in phonology», Phonology Yearbook 7, 193-231.

Ladefoged, Peter, 1989. «Representing phonetic structure», UCLA Working Papers in Phonetcs 73, 1-77.

Ladefoged, Peter et Maddieson, Ian, 1996. The sounds of the world's languages, Oxford, Blackwell.

Liberman, Mark, 1975. The intonational system of English, Cambridge, MIT.

Liberman, Mark et Prince, Alan, 1977. «On stress and linguistic rhythm», Linguistic Inquiry, vol. 8, $\mathrm{n}^{\circ} 2,249-336$.

Liljencrants, Johan et Lindblom, Björn, 1972. "Numerical simulation of vowel quality systems: the role of perceptual contrast», Language 48, 839-862.

Lindblom, Björn, 1986. «Phonetic universals in vowel systems », Ohala, John et Jeager, Jeri (eds.), Experimental phonology, Orlando, Academic Press, 13-44.

Maddieson, Ian, 1984. Patterns of sounds, Cambridge University Press.

Malmberg, Bertil, 1966. Les nouvelles tendances en linguistique, Paris, PUF.

Martinet, André, 1955. Economie des changements phonétiques. Traité de phonologie diachronique, Berne, Francke.

McCarthy, John, 1988. «Feature geometry and dependency: a review», Phonetica 43, 84108.

McCarthy, John et Prince, Alan, 1993. Prosodic morphology: constraint interaction and satisfaction, Amherst, University of Massachusetts.

Mester, Armin, 1986. Studies in tier structure (Ph.D. dissertation), Amherst, University of Massachusetts.

Odden, David, 1991. «Vowel geometry », Phonology 8, 261-289.

- 1994. «Adjacency parameters in phonology», Language, vol. 70, $\mathrm{n}^{\circ} 2,289-330$.

Parkinson, Frederick, 1996. The representation of vowel height in phonology (Ph.D. dissertation), Ohio State University.

Ohala, John, 1975. «Phonetic explanations for nasal sound patterns », Ferguson, Charles et al. (eds.), Nasalfest: Papers from a symposium on nasals and nasalization, Stanford, Language Universals Project, 289-316.

- 1983. «The origin of sound patterns in vocal tract constraints », MacNeilage, Peter (ed.), The production of speech, New York, Springer Verlag, 189-216.

- 1985. «Around Flat», Fromkin, Victoria (ed.), Phonetic Linguistics: Essays in Honor of Peter Ladefoged. Orlando, Academic Press, 223-241.

- 1990. "The phonetics and phonology of aspects of assimilation», Kingston, John et Beckman, Mary (eds.), Papers in Laboratory phonology I. Cambridge University Press, 258-275.

- 1992. «The segment: primitive or derived?», Docherty, Gerard et Ladd, D. R. (eds.), Papers in Laboratory Phonology 2, Cambridge University Press, 166-189.

Ohala, John et Kawasaki, Haruko, 1984. «Prosodic phonology and phonetics », Phonology Yearbook 1, 113-127.

Ohala, John et Lorentz, James, 1977. «The story of [w]: an exercise in the phonetic explanation for sound patterns ", Proceedings of the $3^{\text {rd }}$ Annual Meeting of the Berkeley Linguistic Society, 577-599.

Pierrehumbert, Janet, Beckman, Mary et Ladd, D. R., 2000. «Conceptual foundations of phonology as a laboratory science », Burton-Roberts, Noel et al. (ed.), Phonological Knowledge: Its Nature and Status, Cambridge University Press, 273-303.

Polgárdi, Krisztina, 1998. Vowel harmony. An account in terms of Government and Optimality (Ph.D. dissertation), Leiden University.

Prince, Alan et Smolensky, Paul, 1993. Optimality theory: constraint interaction in Generative grammar, Ms, Rutgers University and University of Colorado, Boulder. 
Ringen, Catherine et Heinämäki, Orvokki, 1999. « Variation in Finnish vowel harmony: an OT account », Natural Language \& Linguistic Theory, vol. 17, $\mathrm{n}^{\circ} 2,303-337$.

Sagey, Elizabeth, 1986. The representation of features and relations in nonlinear phonology, MIT dissertation, Cambridge, Massachusetts.

Sampson, Rodney, 1999. Nasal vowel evolution in Romance, New York, Oxford University Press.

Schane, Sanford, 1984. «The fundamentals of Particle phonology », Phonology Yearbook 1, 129-155.

Scheer, Tobias, 1999. «A theory of consonantal interaction », Folia Linguistica 32, Berlin, Mouton de Gruyter, 201-237.

Selkirk, Elisabeth, 1984. Phonology and syntax: the relation between sound and structure, Cambridge, Massachusetts, MIT Press.

Steriade, Donca, 1987. «Redundant values », Papers from the Annual Regional Meeting of the Chicago Linguistic Society 23, 339-362.

Trigo, Loren, 1991. « On pharynx-larynx interactions », Phonology 8, 113-136.

Troubetzkoy, Nikolaï, 1986. Principes de phonologie, trad. de Grundzüge der Phonologie (1939) par Jean Cantineau, Paris, Editions Klincksieck.

van der Hulst, Harry, 1993. «Les atomes de la structure segmentale : composants, gestes et dépendance », Laks, Bernard et Rialland, Annie (dir.), Architecture des représentations phonologiques, Paris, Éditions du CNRS, 255-290.

Yip, Moira, 1989. "Feature geometry and cooccurrence restrictions», Phonology 6, 349-374. 